\title{
3D Computational Fluid Dynamics of a Treated Vertebrobasilar Giant Aneurysm: A Multistage Analysis
}

\author{
F. Graziano, V.M. Russo, W. Wang, D. Khismatullin, and A.J. Ulm III
}

\begin{abstract}
BACKGROUND AND PURPOSE: The treatment of giant aneurysms of the vertebrobasilar junction remains a challenging task in neurosurgical practice, and the reference standard therapy is still under debate. Through a detailed postmortem study, we analyzed the hemodynamic factors underlying the formation and recanalization of an aneurysm located at this particular site and its anatomic configuration.
\end{abstract}

METHODS: An adult fixed cadaveric specimen with a known VBJ GA, characterized radiographically and treated with endovascular embolization, was studied. 3D computational fluid dynamic models were built based on the specific angioarchitecture of the specimen, and each step of the endovascular treatment was simulated.

RESULTS: The 3D CFD study showed an area of hemodynamic stress (high wall shear stress, high static pressure, high flow velocity) at the neck region of the aneurysm, matching the site of recanalization seen during the treatment period.

CONCLUSIONS: Aneurysm morphologic features, location, and patient-specific angioarchitecture are the principal factors to be considered in the management of VBJ giant aneurysms. The 3D CFD study has suggested that, in the treatment of giant aneurysms, the intra-aneurysmal environment induced by partial coil or Onyx embolization may lead to hemodynamic stress at the neck region, potentially favoring recanalization of the aneurysm.

ABBREVIATIONS: CFD = computational fluid dynamics; $G A=$ giant aneurysm; $V A=$ vertebral artery; $\mathrm{VB}$ J = vertebrobasilar junction; WSS = wall shear stress

G ant aneurysms located at the vertebrobasilar junction are rare and complex lesions that require a multidisciplinary approach. The natural history of these lesions is very poor, and the high risk for rupture, related to the size, makes their treatment mandatory. ${ }^{1-4} \mathrm{On}$ the other hand, the management of these complex entities is extremely challenging and is associated with high rates of morbidity and mortality. ${ }^{5}$ Several treatment options are available nowadays, including either surgical or endovascular procedures. ${ }^{6-8}$ Different factors may play a critical role in the selection of the most appropriate treatment strategy, including preoperative clinical and neurologic status of the patient, evaluation of the features of the aneurysm, re-

Received June 16, 2012; accepted after revision August 25.

From the Clinica Neurochirurgica (F.G., V.M.R.), AOU "G. Rodolico" Universita' degli Studi di Catania, Italy; Department of Neurosurgery (F.G., V.M.R., A.J.U. III), Louisiana State University, New Orleans, Louisiana; and Departments of Chemical and Biomolecular Engineering (W.W.) and Biomedical Engineering (D.K.), Tulane University, New Orleans, Louisiana.

Please address correspondence to Francesca Graziano, MD, Louisiana State University, Department of Neurosurgery, Health Science Center, 2020 Gravier St, 336A, New Orleans, LA; E-mail: 82frensis82@libero.it or 82frensis82@libero.it

- Indicates open access to non-subscribers at www.ajnr.org

http://dx.doi.org/10.3174/ajnr.A3373 lated vascular structures, and collateral circulation. ${ }^{9-15}$ The reference standard therapy remains still under debate, and deep understanding of the hemodynamic engineering of giant aneurysms may provide valuable insight into their behavior and in planning the most effective and successful therapy. ${ }^{16-21}$

\section{MATERIALS AND METHODS}

An extensive postmortem examination of a fixed adult cadaveric specimen with a VBJ giant aneurysm is presented. The aneurysm was characterized radiographically by DSA and CT angiography and was treated with endovascular embolization. First, a 4.5$\mathrm{mm} \times 20-\mathrm{mm}$ Neuroform stent (Boston Scientific, Natick, Massachusetts) was placed across the neck of the aneurysm. One month later, the aneurysm was filled with 11 Guglielmi detachable coils-18 (Boston Scientific) (Fig $1 A-D$ ). Then, 5 months later, recanalization of the aneurysm occurred (Fig $1 E$ ). The coil mass was compacted and shifted to the superior portion of the dome. There was a filling, measuring $40 \times 35 \mathrm{~mm}$, along the right wall of the lesion, in the proximity of the aneurysm neck. The aneurysm was, again, endosaccularly embolized-4 Guglielmi detachable coils-18 were used to create a scaffold inside the re- 

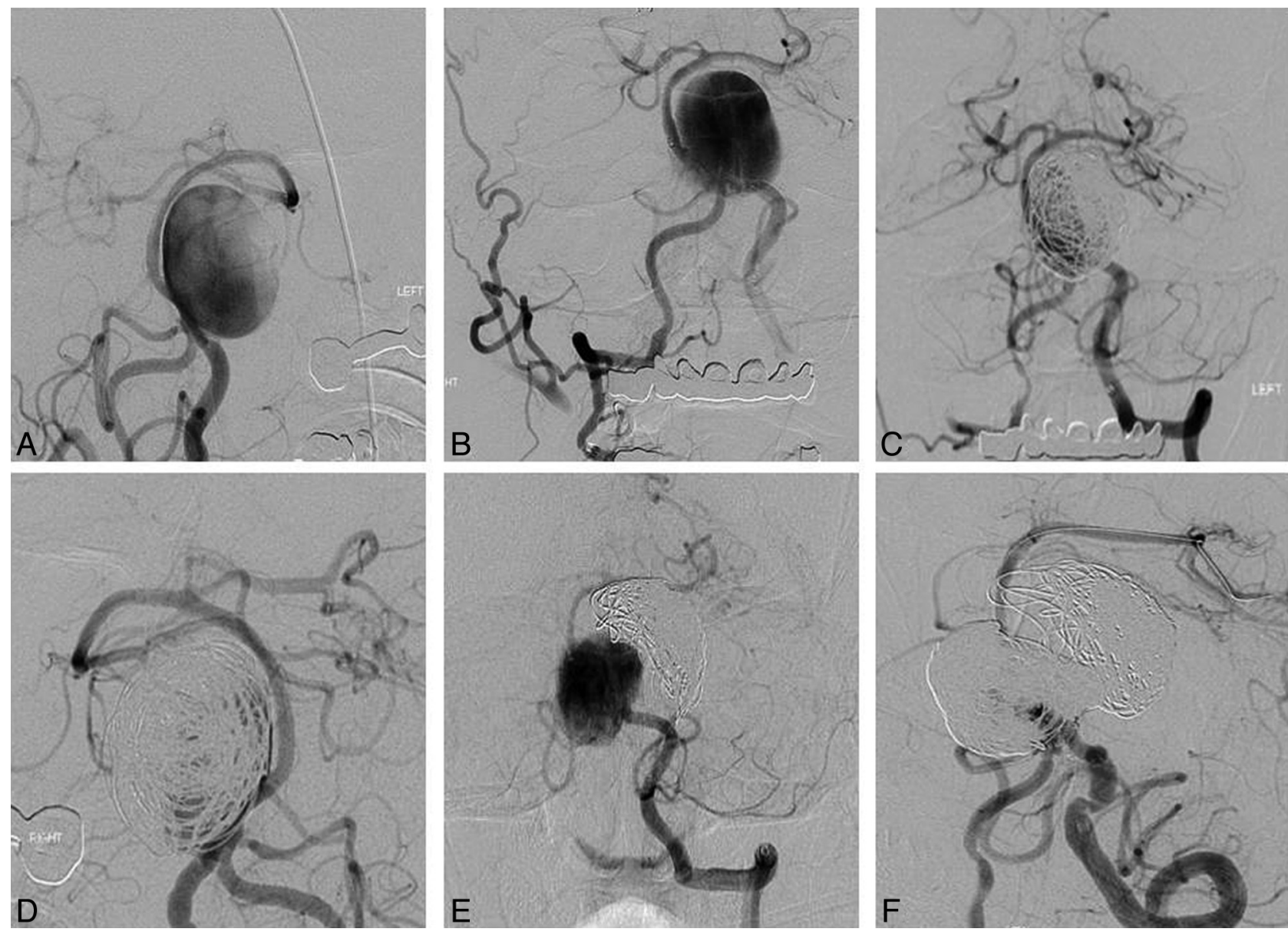

FIG 1. Digital subtraction angiography. A, Left vertebral DSA (lateral view). The giant aneurysm at the VBJ is depicted. The lesion presented an almost spherical shape and a large neck, measuring $14 \mathrm{~mm}$. B, Left vertebral DSA (anterior view). The GA shifted the basilar artery to the right side. $C-D$, Endovascular treatment, Part 1. Left vertebral DSA (lateral view). E-F, Aneurysm recanalization and endovascular treatment, Part 2. F, Left vertebral DSA (anterior view).

canalized part of the aneurysm, and a total of $5 \mathrm{~mL}$ of Onyx 34 (ev3, Irvine, California) and $4 \mathrm{~mL}$ of Onyx 18 (ev3) were injected (Fig $1 F$ ). In addition, the right nondominant vertebral artery was occluded below the PICA origin with the use of Onyx 34. Fourteen months later, the patient died after clinical and neurologic progressive deterioration. In our laboratory investigation, through an analysis of the anatomic aspect of the angioarchitecture of the lesion, 3D CFD simulations of different stages of the endovascular treatments were performed.

\section{Numeric Models}

The 3D CFD models were built in SolidWorks 2008 (SolidWorks, Concord, Massachusetts) on the basis of the specific angioarchitecture of the specimen. The vertebrobasilar system, including the aneurysm, was removed from the specimen, and digital pictures from different angles of view were taken. The digital images were imported into SolidWorks, and the 3D geometry of the models was then built by use of the features of the software "Sweep" and "Loft." In the simulated models, we studied the streamlines, velocity magnitude, blood viscosity, wall pressure, and wall shear stress distributions.

In the digital reconstruction, the vertebrobasilar system was built including both the vertebral arteries (as inlet) and both the
PICA (as outlet) and the basilar artery, until the upper segment, near the bifurcation. Five models of the aneurysm were simulated numerically, each representing a different stage of the endovascular treatment (Fig 2).

The first model corresponded with the outset aneurysm (Fig $2 A)$. The second and third models represented the first endovascular treatment (Fig $2 B-C$ ). In particular, the second model corresponded at the early phase of the embolization process, where the dome of the aneurysm was filled with coils (without blood clots), and the neck region contained blood flow (Fig 2B). As performed elsewhere, we modeled the coiled region as porous medium material ${ }^{22,23}$ because the coils form a fiber matrix as a porous medium material, in which the porosity (fluid volume fraction) depends on the spaces between the fibers (coils). With the assumption that the fibers are randomly distributed in the space, filtration of blood through a coil-filled aneurysm can be described by Darcy's law:

$$
\nabla p=-\frac{\mu}{\alpha} \mathrm{v}
$$

The permeability $\alpha$ of this region can be derived from the empiric equation concluded by Jackson and James, ${ }^{24}$ which is based on the experimental results of randomly distributed fibers made of dif- 


\begin{tabular}{llllll|}
\hline & \\
\end{tabular}

FIG 2. Numeric models of the VBJ GA. A, Outset GA with blood flow (red area). B-C, Endovascular treatment, Part 1. B, Early phase of the embolization treatment. The dome of the aneurysm was filled with coils (through the porous medium material; yellow area) and the neck region with blood flow (red area). C, Late phase of embolization process. The model was divided into 3 regions: 1) a superior half, with attenuated packing of coils (black area); 2) an inferior half (yellow area) with less packing of coils; and 3) the neck region (red area), with blood flow. D-E, Aneurysm recanalization and endovascular treatment, Part 2. The model of the GA was equally increased to $10 \%$. These models have been simulated as being completely filled by a solid material (black region), except at the neck region. $E$, The model was built without the right VA.

ferent materials. The number and size of coils simulated in this study corresponded to those used in the actual therapy (Fig 2).

In the third model, we simulated the late phase of the endovascular embolization in which the aneurysm was divided, for the sake of simplicity, in 3 regions: 1 ) a superior half, with an attenuated coil packing, as depicted in our angiography study; 2) an inferior half, which, during the endovascular treatment, was filled with less packing of coils to allow for perfusion of the perforating branches; and 3) the neck region, with blood flow. In the superior half, we simulated the formation of blood clots in this late phase of the embolization process (Figs $1 C,-D$ and $2 C$ ). The blood clots are viscous-elastic materials and are less deformable than blood but can still be deformed under stress. So far, none of the CFD simulations have taken into account the role played by blood clots in changes of flow field because no reliable measurements of their mechanical properties are available. In our study, the blood clots were assumed to be a solid material, though the lack of complete faithfulness to the real case was recognized. The inferior half of the aneurysm was still simulated with a discrete porosity, and the porous medium material was again applied (Figs $1 C-E$ and $2 C$ ).

In the fourth and fifth models, recanalization of the aneurysm was simulated. As shown in the angiographic study, the recanalized portion $(40 \times 35 \mathrm{~mL})$ corresponded to $10 \%$ of the initial volume of the aneurysm $(15.5 \mathrm{~mL})$. To numerically reproduce this stage, we proportionally increased the model to $10 \%$ of volume (Fig 2D, -E). These models represented the second endovascular treatment, in which the liquid embolic agent (Onyx) was also used to obliterate the recanalized region. The Onyx becomes solid once it comes into contact with blood; therefore, it was simulated as a solid material. In these models, the aneurysm was assumed to be completely filled by a solid material, except at the neck region (Fig 2D). The fifth model represented the final stage of the second treatment, in which the right vertebral artery was occluded. Therefore, the model was built without this vessel (Fig 2E).

\section{Flow Field Features}

The flow fields in blood vessels were numerically simulated with the CFD software FLUENT 12.0 (ANSYS, Lebanon, New Hampshire). In our models, blood flow was modeled as non-Newtonian with shear-dependent dynamic viscosity. ${ }^{25}$ This property was captured by use of the Carreau constitutive equation, largely used to describe the shear thinning property of human blood. ${ }^{26}$ The flow was assumed to be laminar (because the Reynolds number based on the vessel diameter and maximal flow velocity, $0.25 \mathrm{~m} / \mathrm{s}$, of the basilar artery was $\operatorname{Re}=\rho U D / \mu_{\propto} \approx 300$, which was not enough to determine turbulence) and incompressible, with an attenuation of $1050 \mathrm{~kg} / \mathrm{m}^{3}$. All of the inlets were defined as mass-flow inlets on which the mass-flow rate was defined so that the main flow rate in the basilar artery was $200 \mathrm{~mL} / \mathrm{min}$ (Fig 2). ${ }^{20,27}$ All of the outlets were defined as the outflow boundaries on which the diffusion flux for any variable was zero. Either for the inlets or for the outlets, the flow rates were distributed proportionally to the corresponding cross-sectional areas of the inlet or outlet boundaries. Because patient-specific blood flow information was not available, a nonpulsatile steady-state flow was assumed. The vessel walls were simplified as noncompliant walls without any flexibility. With these assumptions, the governing equations were the steady-state incompressible Navier-Stokes equations: 


$$
\begin{gathered}
" \nabla \cdot \mathrm{v}=0 \\
\rho \mathrm{v} \cdot \nabla \mathrm{v}=-\nabla p+\mu \nabla^{2} \mathrm{v},
\end{gathered}
$$

The computational domain was discretized with hybrid unstructured meshes. Specifically, a tetrahedral mesh was used in the bulk region of the domain, and 5 layers of the boundary layer mesh were added to accurately describe the velocity gradients near the vessel wall. The thickness of the first boundary layer was $0.02 \mathrm{~mm}$, and the total height of the boundary layer mesh was $0.2 \mathrm{~mm}$.

\section{RESULTS}

\section{Model I: Outset Aneurysm}

In the outset aneurysm, the blood flow formed rotating vortices (Fig 3A.1). They were most visible at the inflow zone of the aneurysm because, while progressing in the lesion, they enlarged and lost their cohesion. The blood flow presented a higher velocity magnitude at the superior half of the aneurysm, close to the wall, whereas the central area was characterized by a stagnant flow (Fig 3B.1). The highest velocity of the blood flow was found at the neck region. The apparent viscosity at the central area of the aneurysm was elevated, but it was very low at the neck region (Fig 3C.1). Because the diameter of the left VA was larger than that of the right VA ( $4.25 \mathrm{~mm}$ and $1.47 \mathrm{~mm}$, respectively), the flow rate coming from the left VA was much larger than that coming from the right VA $(266 \mathrm{~mL} / \mathrm{min}$ and $54 \mathrm{~mL} / \mathrm{min}$, respectively), and the blood flow was scouring the right side of the aneurysm more than the left side. Hot spots of high static pressure and WSS were observed on the anteromedial surface of the right half of the aneurysm, in proximity to the VBJ (Fig $4 A .1,-B .1$ ).

\section{Model II: Aneurysm Filled by Coils (First Endovascular Treatment, "Early Phase")}

The total volume of coils used during the endovascular procedure was $0.26 \mathrm{~mL}$, and the solid volume fraction was $\phi=1.7 \%$. Thus, only $1.7 \%$ of the total volume of the aneurysm $(15.5 \mathrm{~mL})$ was filled by coils (Fig 2). In our model, the porous medium material was used to simulate the attenuation of the coils inside the aneurysm; this provided a reduction in the swirling structure and velocity magnitude of the blood flow. Although coils occupied only a small part of the aneurysm, they induced significant flow disturbances (Fig 3A.2). In the superior half of the aneurysm, close to the wall, the velocity of the blood flow decreased; in the central area of the dome, it was completely stagnant. In this area also, blood viscosity was increased (Fig 3B.2, -C.2). At the neck region, high flow velocity was still present, and blood viscosity remained unchanged (Fig 3B.2, -C.2). The hot spots of static pressure and WSS decreased along the posteromedial surface of the right half of the aneurysm, but at the neck region, the hemodynamic stress was found to be constantly high (Fig 4A.2, -B.2).

\section{Model III: Aneurysm Filled with Coils and Blood Clots (First Endovascular Treatment, "Late Phase")}

The late phase is characterized by the formation of blood clots in the area of the aneurysm with a high packing attenuation of coils, as seen in the angiographic study. The formation of blood clots, simulated as solid material in the superior half of the aneurysm, caused a decrease in blood flow at this region (Fig 3A.3). The vortex structure of the flow was squeezed and limited to the inferior half and neck region of the aneurysm. In the remaining inferior half (simulated as being filled with coils through the porous medium material), the velocity magnitude of the blood flow was slightly increased, with reduced viscosity, but it remained constantly high at the neck region (Fig 3B.3, -C.3). Hot spots of static pressure and WSS moved to the posteromedial surface of the right half of the aneurysm and to the neck region, and the values increased (Fig 4A.3, -B.3).

\section{Models IV and V: Aneurysm Recanalized, Filled Completely with Blood Clots and Onyx (Second Endovascular Treatment)}

In these models, the size of the aneurysm was proportionally increased by $10 \%$ of the initial volume. The dome of the aneurysm was simulated as being completely filled by solid material because both the blood clots and Onyx were modeled without porosity. The blood flow inside the aneurysm was then reduced to a value of zero (Fig $3 A .4,-A .5$ ). Velocity magnitude of the blood flow at the neck of the aneurysm was high, and blood viscosity was very low (Fig 3B.4, - B.5; 3C.4, -C.5). At this region, the hot spots of static pressure and WSS were larger and the values high (Fig 4A.4, -B.4). In the fifth model, we simulated the occlusion of the right vertebral artery. Because its blood flow rate was calculated as being one-fifth of the flow rate in the left VA, the suppression of the flow within the vessel did not provide particular changes in the flowfield structure, except for a slight enlargement of the area with hemodynamic stress at the neck region (Fig 4A.5, -B.5).

\section{DISCUSSION}

Recently, cerebrovascular diseases have become a point of interest in biomedical engineering research. The role of WSS in cytologic changes of the arterial walls and the variable geometry of the arterial system have been found to be critical in the onset of vascular diseases, such as aneurysmal lesions or atherosclerotic phenomena. ${ }^{16,20,21,28-34}$ The role of WSS is still controversial in the development and growth of cerebral aneurysms ${ }^{16-18}$; in fact, both excess and lack of WSS can lead to pathologic phenomena that cause changes in the biomechanical properties of the arterial wall. ${ }^{29}$ The presence of a low-flow environment leads to regions of low WSS $(<0.4 \mathrm{~Pa})$, which can be detrimental to the vessel endothelium. The formation of thrombi promoted by stagnant blood flow can lead to the release of substances that promote inflammation and degradation of the aneurysmal wall, with endothelial proliferation and apoptosis, favoring aneurysmal growth and rupture. ${ }^{29,32,35,36}$ By contrast, high WSS, which is associated with high blood flow, can lead to dysfunction of the endothelium, with progressive deformation of the wall until rupture occurs. ${ }^{31,37} \mathrm{Meng}$ et $\mathrm{al}^{38} \mathrm{re}$ cently demonstrated in an animal model that high WSS contributes to aneurysmal initiation and development. They found that areas with high WSS and high WSS spatial gradients were associated with remodeling of the wall of an aneurysm, such as disrupted internal elastic lamina and endothelium, thinned media, and smooth muscle cell loss. Cebral et $\mathrm{al}^{17,18}$ reported that the flow pattern and impingement region, defined as the region of the aneurysm where the inflow stream affects the aneurysmal wall with high WSS, correlate with rupture of the aneurysm. In partic- 


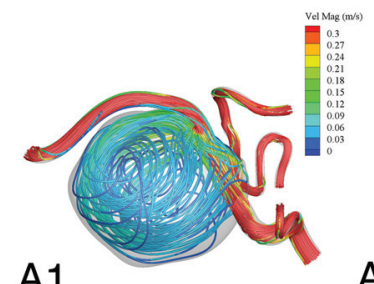

A1

$\mathrm{A} 2$
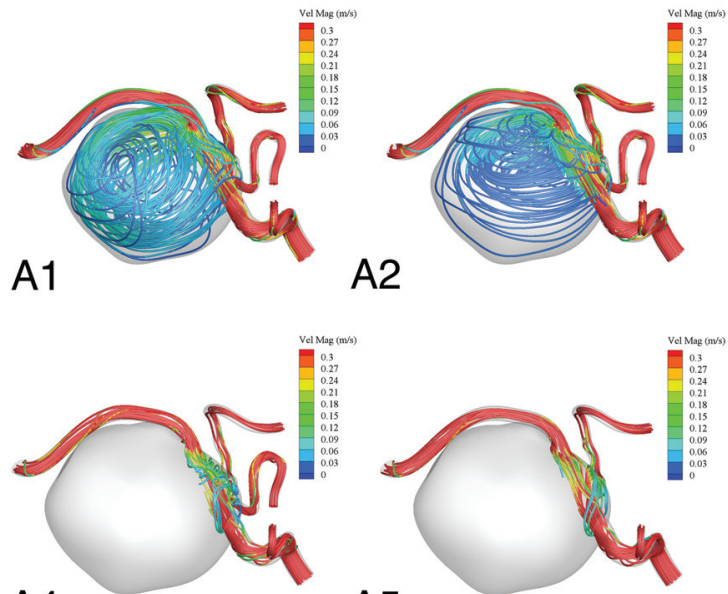

A4

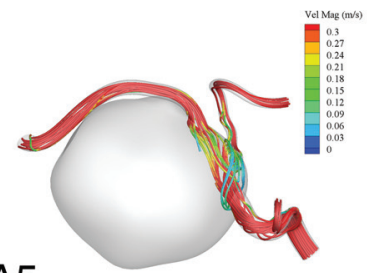

A5

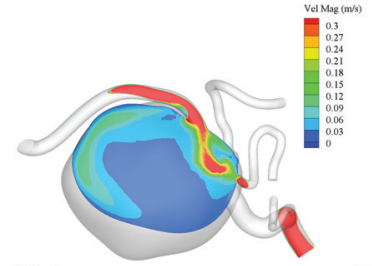

B1

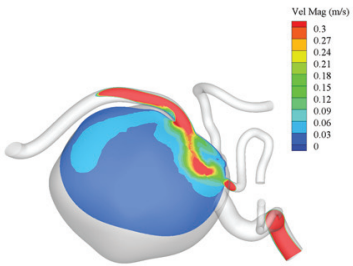

B2

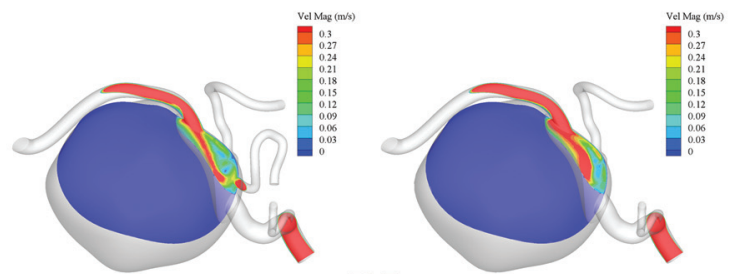

B4

B5
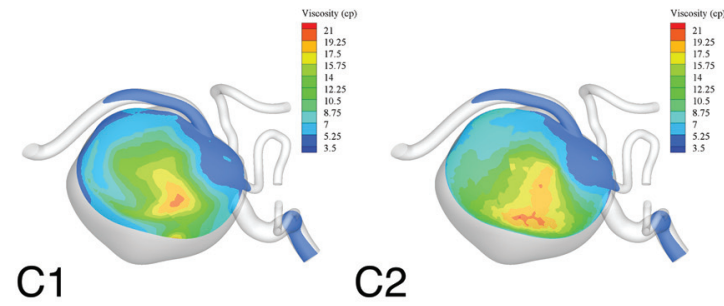

C2

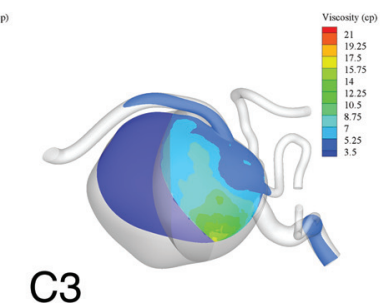

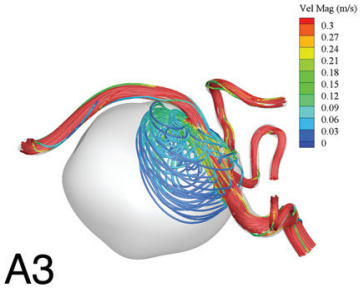

A3

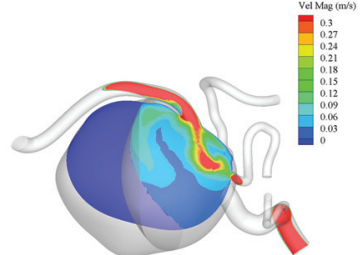

B3

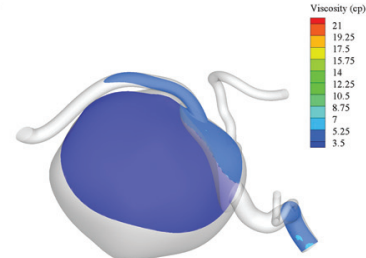

C5

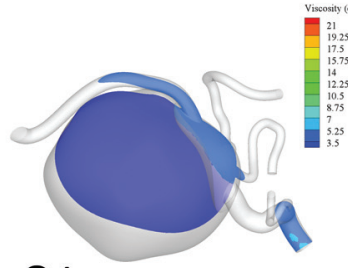

C4

FIG 3. Streamlines, velocity magnitude, and viscosity distributions. A, Streamlines (lateral views). Al, The blood flow formed rotating vortices at the inflow zone. $A 2$, The attenuation of the coils inside the aneurysm provided a reduction of the swirling structure of the blood flow. $A 3$, The vortex structure of the flow was squeezed and limited to the inferior half and to the neck region of the aneurysm. $A 4$ and $A 5$, Blood flow inside the aneurysm was reduced to a value of zero. No flow changes occurred with the suppression of the right VA. $B$, Velocity magnitude (cross-sectional views). B1, The highest velocity of the blood flow was found at the neck region. B2, Although coils occupied only a small part of the aneurysm, they induced significant disturbances in flow. B3, The velocity magnitude of the blood flow remained constantly high at the neck region. $B 4$ and $B 5$, These models have been simulated as being completely solid; therefore, the velocity magnitude was zero. $C$, Viscosity (cross-sectional views). Cl, The apparent viscosity at the central area was elevated, whereas it was very low at the neck region. C2, The viscosity of the blood was increased in the central area. C3, The inferior half of the aneurysm presented a decreased viscosity. C4 and C5, In the neck region, where blood flow was still simulated, a very low viscosity was seen. 


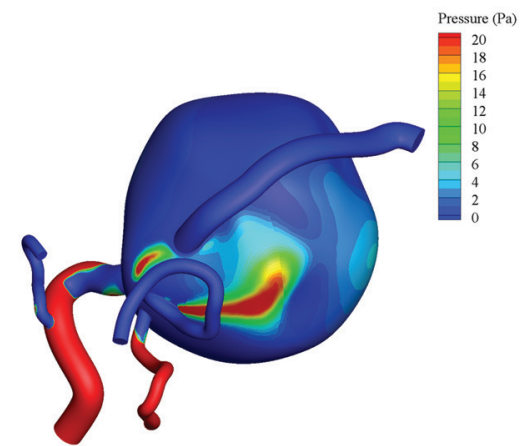

A1

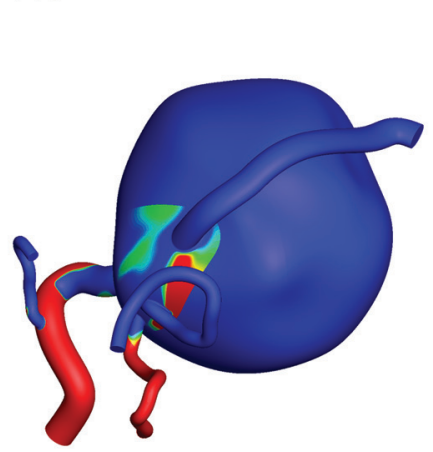

A4

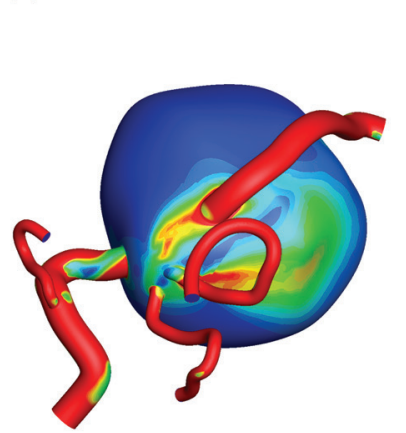

B1
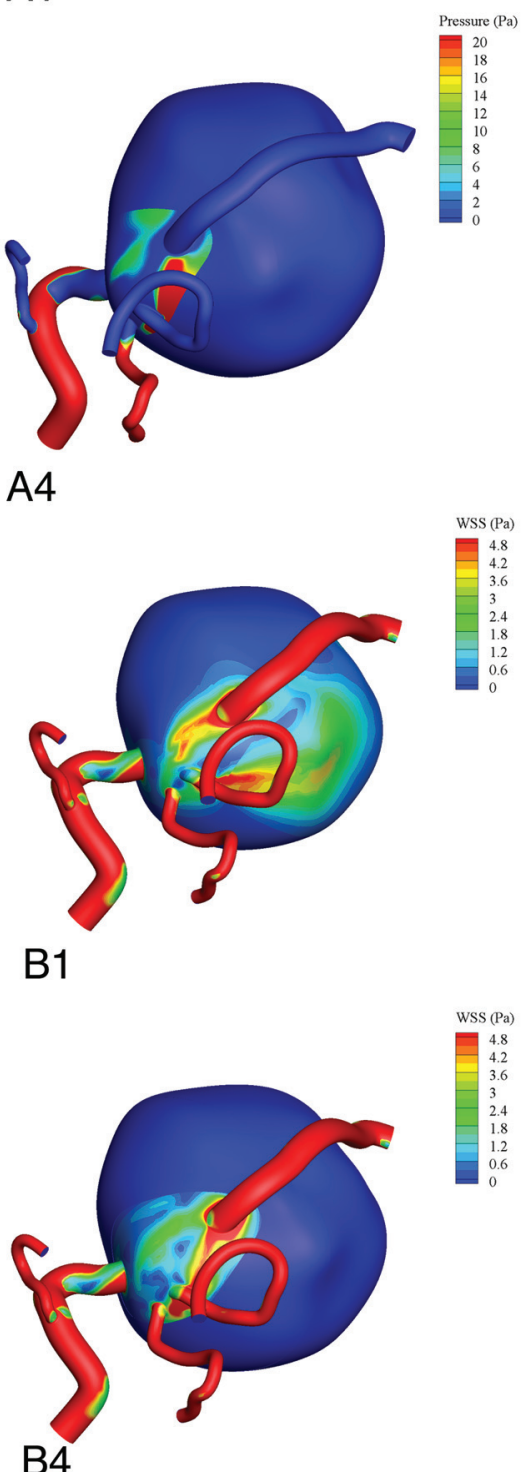

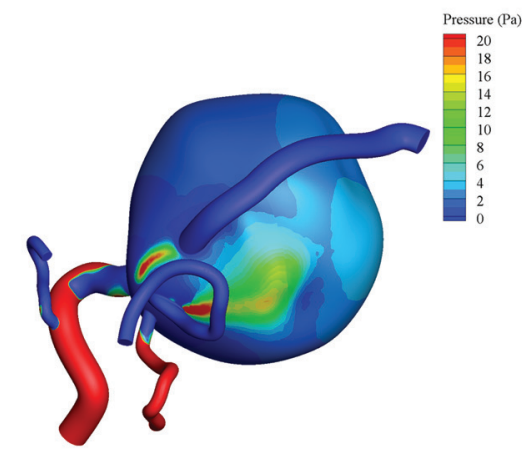

A2

A5
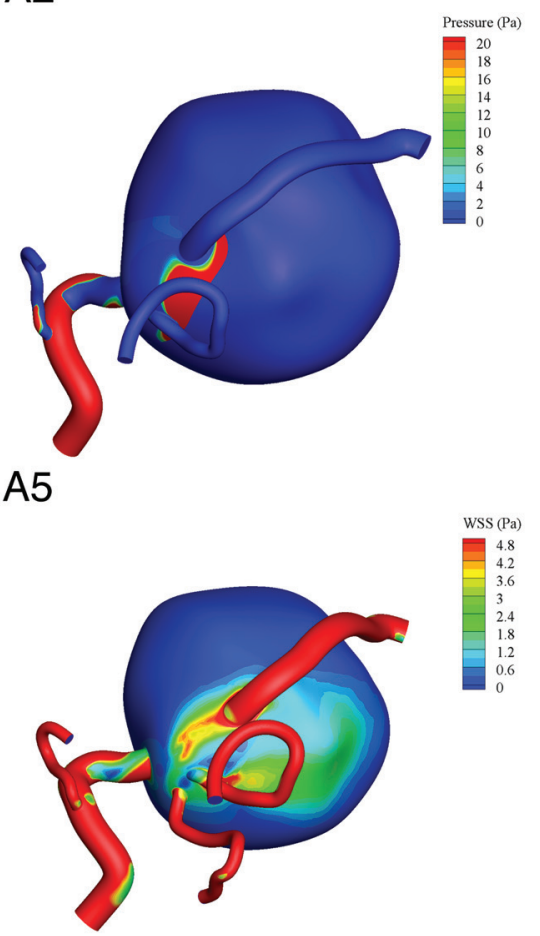

B2
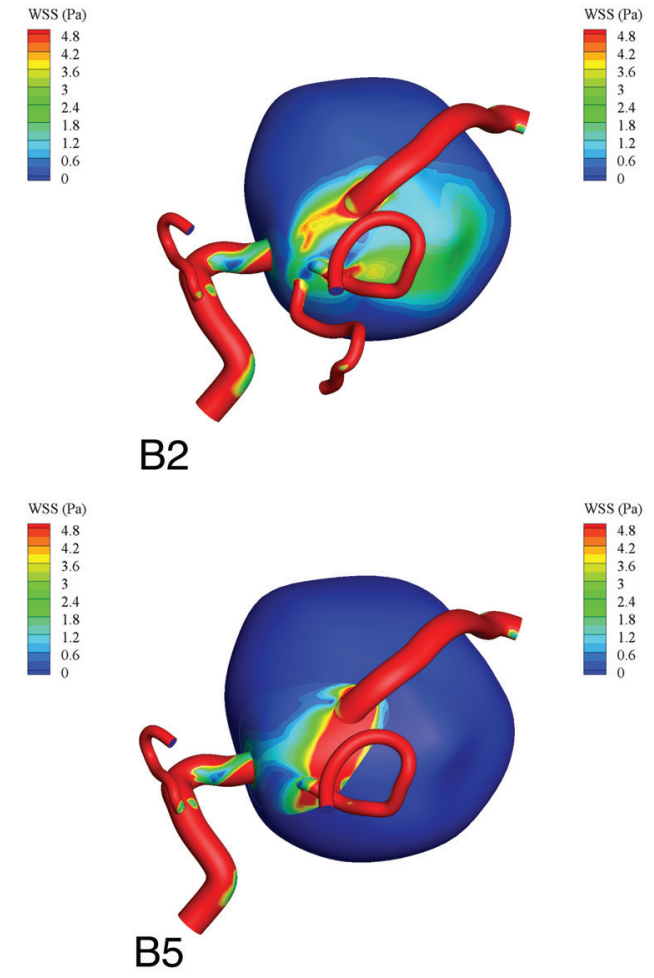

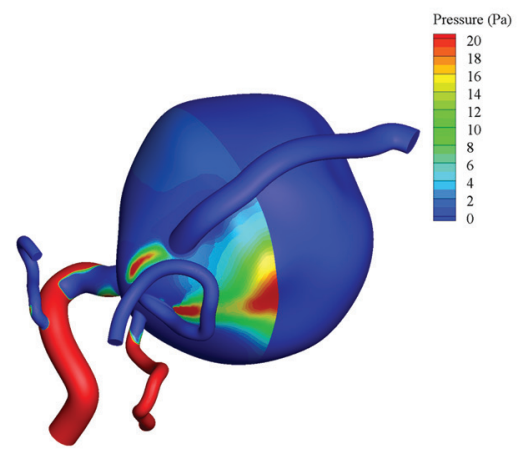

A3

FIG 4. Static pressure and wall shear stress distributions. $A 7$ and $B 1$, Hot spots of high static pressure and WSS were observed on the posteromedial surface of the right half of the outset aneurysm, in proximity to the VBJ. A2 and B2, The hot spots of static pressure and WSS decreased along the posteromedial surface of the right half of the aneurysm. $A 3$ and $B 3$, The hot spots of static pressure and WSS moved to the posteromedial surface of the right half of the aneurysm and to the neck region, and their values increased. $A 4$ and $A 5$ and $B 4$ and $B 5$, At the neck region, the hot spots of static pressure and WSS were larger and higher.

ular, a disturbed flow pattern, a small impingement region, and narrow jets were associated with this event. Utter and Rossmann ${ }^{39}$ supported the thesis that progressive changes in the shape of the aneurysm occur after an initial injury, secondary to an excessive
WSS on the endothelial cells. These changes generate a progressive decrease of WSS and lead to endothelial dysfunction, wall remodeling, and aneurysm growth.

In the vertebrobasilar system, the presence of a symmetric 
structure at the junction provides a regular velocity profile, with blood flow running parallel to the vessel wall. This profile lowers the hemodynamic stress at the VBJ, accounting for the relatively low incidence of aneurysm formation at this site. However, when the diameter of the VAs is very different or the basilar artery is badly bent, the flow in the basilar artery is disturbed as a result of swirling flows at the $\mathrm{VBJ} .{ }^{20}$ When an aneurysm is located at the VBJ and the VAs present a different diameter, the major flow comes from the vessel with the larger diameter. The jet flow hits 1 side of the inflow zone of the aneurysm with great force, leading to histopathologic changes and deformations in the arterial wall. ${ }^{40}$

In the specific angioarchitecture of the specimen studied, the diameters of the VAs were significantly different, with a different flow rate (Fig 2). The asymmetric configuration of the vertebrobasilar system generated a sharpened velocity profile at the VBJ and increased hemodynamic stress, which could be considered as factors promoting the formation of the aneurysm at this site (Fig $3 A .1,-B .1$ and $4 A .1,-B .1)$.

The effect of coil packing of the aneurysm through simulation of the endovascular treatment was also investigated (Fig 2B, $-C$ ). Other studies ${ }^{41-44}$ have reported that when the aneurysm is not tightly occluded, the coils have a propensity to gather together, being pushed and displaced toward the dome by the arterial pulsatile flow ("water-hammer effect"). The volumetric ratio of coils in the treatment of small aneurysms has been previously calculated. The minimal attenuated packing to obtain an intra-aneurysmal thrombosis has been estimated to be approximately $26 \%-$ $33 \%$; the maximal attenuated packing achievable without the risk for coil migration was roughly $30 \%-36 \% .{ }^{45}$ In our study, the total coil volume was $0.26 \mathrm{~mL}$, and the solid volume fraction was $\phi=$ $1.7 \%$. This finding shows that, to reach the minimal volumetric ratio of coils, a large quantity of coils (approximately $80 \mathrm{~m}$ in our study) is needed when giant aneurysms are treated. The hemodynamic environment created by the coiling procedure has facilitated intra-aneurysmal thrombosis and prevented the risk for rupture because the flow was stagnant and the pressure on the aneurysm wall was decreased. On the other hand, the redistribution of the wall pressure and the increased WSS and flow velocity at the neck region (wide and not embolized to preserve the perfusion of the perforating vessels) have favored the coil compaction and increased the risk for recanalization at this region (Fig $3 A-C, 4 A .3$, and $4 B .3){ }^{18,46-48}$

Although it has been demonstrated that the occlusion of a single VA can affect the flow-field structure at the VBJ, ${ }^{35}$ in our study, the occlusion of the right VA was not associated with important flow changes because the flow rate coming from this vessel was much smaller than the flow rate coming from the contralateral VA.

\section{CONCLUSIONS}

Vertebrobasilar giant aneurysms pose extreme challenges in neurosurgical practice. The accurate selection of the operative strategy is the key to safe treatment with a long-term benefit; 3D CFD simulations may represent a valuable adjunctive resource that, coupled with the standardized preoperative radiologic work-up, could be able to tailor the most effective treatment.
Disclosures: Francesca Graziano-RELATED: Provision of Writing Assistance, Medicines, Equipment, or Administrative Support: Louisiana State University, New Orleans. * Vittorio Russo-RELATED: Support for Travel to Meetings for the Study or Other Purposes: Louisiana State University, New Orleans. * Arthur Ulm-UNRELATED: Employment: HW Neurological Institute, Nashville, Tennessee. *Money paid to institution. Disclaimer: The authors report no conflict of interest concerning the materials or methods used or the findings specified in this study.

\section{REFERENCES}

1. Drake CG. The treatment of aneurysms of the posterior circulation. Clin Neurosurg 1979;26:96-144

2. MacFarlane MR, McAllister VL, Whitby DJ, et al. Posterior circulation aneurysms. Results of direct operations. Surg Neurol 1983;20:399-413

3. Vertebrobasilar aneurysms. In: Yasargil M, Smith RD, Young PH, et al. Microneurosurgery in 4 Volumes. New York: Georg Thieme Verlag; 1986;232-95

4. Tiyaworabun S, Wanis A, Schirmer M, et al. Aneurysms of the vertebro-basilar system: clinical analysis and follow-up results. Acta Neurochir (Wien) 1982;63:221-29

5. Unruptured intracranial aneurysms-risk of rupture and risks of surgical intervention. International Study of Unruptured Intracranial Aneurysms Investigators. N Engl J Med 1998;339:1725-33

6. Gonzalez LF, Amin-Hanjani S, Bambakidis NC, et al. Skull base approaches to the basilar artery. Neurosurg Focus 2005;19:E3

7. Hanel RA, Spetzler RF. Surgical treatment of complex intracranial aneurysms. Neurosurgery 2008;62:1289-97; discussion 1297-99

8. Spetzler RF, Riina HA, Lemole GM Jr. Giant aneurysms. Neurosurgery 2001;49:902-08

9. Drake CG. Ligation of the vertebral (unilateral or bilateral) or basilar artery in the treatment of large intracranial aneurysms. J Neurosurg 1975;43:255-74

10. Lawton MT, Hamilton MG, Marcos JJ, et al. Revascularization and aneurysm surgery: current techniques, indications, and outcome. Neurosurgery 1996;38:83-92; discussion 92-94

11. Lawton MT, Spetzler RF. Surgical strategies for giant intracranial aneurysms. Acta Neurochir Suppl, 1999;72:141-56

12. Lemole GM Jr, Spetzler RF, Riina HA. Giant aneurysms. In: Winn HR, Berger MS, Dacey RG Jr. Youmans Neurological Surgery. Philadelphia: Saunders; 1996;2079-2100

13. Peluso JP, van Rooij WJ, Sluzewski M, et al. Aneurysms of the vertebrobasilar junction: incidence, clinical presentation, and outcome of endovascular treatment. AJNR Am J Neuroradiol 2007;28: 1747-51

14. Pelz DM, Viñuela F, Fox AJ, et al. Vertebrobasilar occlusion therapy of giant aneurysms. Significance of angiographic morphology of the posterior communicating arteries. J Neurosurg 1984;60: $560-65$

15. Solomon RA, Smith CR, Raps EC, et al. Deep hypothermic circulatory arrest for the management of complex anterior and posterior circulation aneurysms. Neurosurgery 1991;29:732-37; discussion 737-38

16. Boussel L, Rayz V, McCulloch C, et al. Aneurysm growth occurs at region of low wall shear stress: patient-specific correlation of hemodynamics and growth in a longitudinal study. Stroke 2008;39: 2997-3002

17. Cebral JR, Castro MA, Burgess JE, et al. Characterization of cerebral aneurysms for assessing risk of rupture by using patient-specific computational hemodynamics models. AJNR Am J Neuroradiol 2005;26:2550-59

18. Cebral JR, Mut F, Weir J, et al. Association of hemodynamic characteristics and cerebral aneurysm rupture. AJNR Am J Neuroradiol 2011;32:264-70

19. Groden C, Laudan J, Gatchell S, et al. Three-dimensional pulsatile flow simulation before and after endovascular coil embolization of a terminal cerebral aneurysm. J Cereb Blood Flow Metab 2001;21:1464-71

20. Kobayashi N, Karino T. Flow patterns and velocity distributions in 
the human vertebrobasilar arterial system. Laboratory investigation. J Neurosurg 2010;113:810-19

21. Nixon AM, Gunel M, Sumpio BE. The critical role of hemodynamics in the development of cerebral vascular disease. J Neurosurg 2010;112:1240-53

22. Mitsos AP, Kakalis NM, Ventikos YP, et al. Haemodynamic simulation of aneurysm coiling in an anatomically accurate computational fluid dynamics model: technical note. Neuroradiology 2008;50:341-47

23. Gruber A, Killer M, Bavinzski G, et al. Clinical and angiographic results of endosaccular coiling treatment of giant and very large intracranial aneurysms: a 7-year, single-center experience. Neurosurgery 1999;45:793-803; discussion 803-04

24. Jackson GW, James DF. The permeability of fibrous porous media. Can J Chem Eng 1986;64:364-74

25. Gonzalez CF, Cho YI, Ortega HV, et al. Intracranial aneurysms: flow analysis of their origin and progression. AJNR Am J Neuroradiol 1992;13:181-88

26. Shibeshi SS, Collins WE. The rheology of blood flow in a branched arterial system. Appl Rheol 2005;15:398-405

27. Cebral JR, Castro MA, Putman CM, et al. Flow-area relationship in internal carotid and vertebral arteries. Physiol Meas 2008;29:585-94

28. Hoi Y, Meng H, Woodward SH, et al. Effects of arterial geometry on aneurysm growth: three-dimensional computational fluid dynamics study. J Neurosurg 2004;101:676-81

29. Shojima M, Oshima M, Takagi $K$, et al. Magnitude and role of wall shear stress on cerebral aneurysm: computational fluid dynamic study of $\mathbf{2 0}$ middle cerebral artery aneurysms. Stroke 2004;35: 2500-05

30. Singh PK, Marzo A, Howard B, et al. Effects of smoking and hypertension on wall shear stress and oscillatory shear index at the site of intracranial aneurysm formation. Clin Neurol Neurosurg 2010;112: $306-13$

31. Nakatani H, Hashimoto N, Kang Y, et al. Cerebral blood flow patterns at major vessel bifurcations and aneurysms in rats. J Neurosurg 1991;74:258-62

32. Malek AM, Alper SL, Izumo S. Hemodynamic shear stress and its role in atherosclerosis. JAMA 1999;282:2035-42

33. Acevedo-Bolton G, Jou LD, Dispensa BP, et al. Estimating the hemodynamic impact of interventional treatments of aneurysms: numerical simulation with experimental validation: technical case report. Neurosurgery 2006;59:E429-30; author reply E429-30

34. Takeuchi S, Karino T. Flow patterns and distributions of fluid ve- locity and wall shear stress in the human internal carotid and middle cerebral arteries. World Neurosurg 2010;73:174-85; discussion e27

35. Jou LD, Quick CM, Young WL, et al. Computational approach to quantifying hemodynamic forces in giant cerebral aneurysms. AJNR Am J Neuroradiol 2003;24:1804-10

36. Griffith TM. Modulation of blood flow and tissue perfusion by endothelium-derived relaxing factor. Exp Physiol 1994;79:873-913

37. Sho E, Sho M, Singh TM, et al. Blood flow decrease induces apoptosis of endothelial cells in previously dilated arteries resulting from chronic high blood flow. Arterioscler Thromb Vasc Biol 2001;21: $1139-45$

38. Meng H, Wang Z, Hoi Y, et al. Complex hemodynamics at the apex of an arterial bifurcation induces vascular remodeling resembling cerebral aneurysm initiation. Stroke 2007;38:1924-31

39. Utter B, Rossmann JS. Numerical simulation of saccular aneurysm hemodynamics: influence of morphology on rupture risk. J Biomech 2007;40:2716-22

40. Gonzalez NR, Duckwiler G, Jahan R, et al. Challenges in the endovascular treatment of giant intracranial aneurysms. Neurosurgery 2006;59(5 Suppl 3):S113-24; discussion S3-13

41. Kwan ES, Heilman CB, Shucart WA, et al. Enlargement of basilar artery aneurysms following balloon occlusion-"water-hammer effect". Report of two cases. J Neurosurg 1991;75:963-68

42. Choudhari KA, Flynn PA, McKinstry SC. Spontaneous extrusion of guglielmi detachable coils from anterior communicating artery aneurysm. Neurol India 2007;55:148-50

43. Ahlqvist J. Stress-related intracerebral hemorrhage and the WaterHammer effect. Stroke 2001;32:275-78

44. Sekhar LN, Heros RC. Origin, growth, and rupture of saccular aneurysms: a review. Neurosurgery 1981;8:248-60

45. Piotin M, Mandai S, Murphy KJ, et al. Dense packing of cerebral aneurysms: an in vitro study with detachable platinum coils. AJNR Am J Neuroradiol 2000;21:757-60

46. Grunwald IQ, Papanagiotou P, Struffert T, et al. Recanalization after endovascular treatment of intracerebral aneurysms. Neuroradiology 2007;49:41-47

47. Sforza DM, Putman CM, Scrivano E, et al. Blood-flow characteristics in a terminal basilar tip aneurysm prior to its fatal rupture. AJNR Am J Neuroradiol 2010;31:1127-31

48. Lubicz B, Leclerc X, Gauvrit JY, et al. Giant vertebrobasilar aneurysms: endovascular treatment and long-term follow-up. $\mathrm{Neu}$ rosurgery 2004;55:316-23; discussion 323-26 